\title{
A Quantitative Method for Separation of Semantic Subspaces
}

\author{
Oliver C. S. Tzeng \\ University of Illinois at Urbana-Champaign
}

\begin{abstract}
A new method for separating affective and denotative meaning subsystems in semantic differential ratings of any homogeneous concept domain is developed and illustrated using personality ratings data. Results indicated that Osgood's Evaluation, Potency and Activity dimensions were dominant in the underlying semantic structure of personality conceptions and that four dimensions in the "other" subspace, orthogonal to Affect, were clearly interpretable, "affect-free" descriptive features of personalities. Possible applications of this model to other social and psychological research are discussed.
\end{abstract}

Tzeng (1975a) has argued that human perceiving and judging involves three major variables: unique characteristics of the individuals processing the information (individual differences), characteristics of the objects perceived and judged (signs or assigns of events, personalities, drugs, etc.), and the meaning systems (sets of semantic features) these individuals have developed. The formation of the meaning components is historically dependent (i.e., the experience of the organism) on the other two variables-interaction of humans with objects (including other humans) in their environment.

APPLIED PSYCHOLOGICAL MEASUREMENT

Vol. 1. No. 2 Spring 1977 pp. 171-184

(c) Copyright 1977 West Publishing Co.
Therefore the meanings of the same objects for different individuals will vary to the extent that their behaviors toward the objects have varied. This implies that the meanings of signs will reflect the idiosyncracies of individual learning experiences.

Due to different processes in formulating psychological dispositions, meanings of objects can further be dichotomized into two aspects-affective and denotative (Tzeng, 1975b). According to Osgood (1971), for survival in the evolutionary sense it is crucial for the human animal, as well as other higher organisms, to make different emotional (autonomic) reactions to distinguish among the signs of things as being good or bad (Evaluation), strong or weak (Potency), and active or passive (Activity) with respect to himself when confronting any situation calling for judgment or a behavioral decision. These distinguishing processes reflect a person's feelings about (including attitude toward) an object. They are primarily emotional in nature and thus can be termed an affective meaning system. When meanings of signs are established in terms of individual sensorimotor discrimination systems to characterize objects or events referentially, they reflect a person's implicit judgment or descriptive criteria about the object. The criteria include various conceptual categories, such 
as groupings, contrast, similarity and classification. Meanings from this abstract structure of signs are primarily non-affective in nature, and thus may be defined as part of a denotative meaning system. Typically both the affective and denotative meaning systems are involved simultaneously in human perceptions and judgments. Therefore, measurements of their functional relationships with object variables for each individual are important in the social and behavioral sciences. Furthermore, these two meaning systems, as the underlying semantic criteria of differential cognition, render many diverse objects and overt behaviors functionally equivalent within and even across different cultures. In order to understand the dynamics of intra- and inter-personal behaviors, the identification of affective and denotative semantic features in any particular conceptual (object) domain and their influence in accounting for individual differences may become a prime goal in social and psychological research.

A well-known quantitative technique for measuring certain aspects of the meanings of perceptual and linguistic signs is the Semantic Differential (SD) technique (Osgood, Suci and Tannenbaum, 1957). The SD has been applied to diverse groups of subjects judging diverse sets of concepts (from natural space concepts such as MOON and RIVER to abstractions such as EDUCATION and PEACE) and evidence for three dominant and universal affective dimensions of Evaluation (E), Potency (P), and Activity (A) has been found in both indigenous and cross-cultural studies conducted in some 30 language-culture communities (Osgood, 1971; Osgood, May and Miron, 1975). Given the necessarily metaphorical use of scales in rating 100 or more heterogeneous concepts (e.g., SYMPATHY: hot-cold; TORNADO: fair-unfair; etc.) and their rotations toward their dominant affective features, the three resultant dimensions generally have been assumed to represent purely affective components of human semantic systems (Osgood, 1969). Therefore, under the diversity of naturalistically elicited scales used, the EP-A dimensions would appear to be the maxi- mal number of affective components in the entire semantic space. They seem essentially identical to Wundt's three dimensions of feeling-pleasantness/unpleasantness, tension/relief, and excitement/quiet (Osgood, et al., 1975, Ch. 4).

Tzeng (1972) has argued that when SD judgments are obtained for a set of more homogeneous concepts (e.g. all relating to personalities), the affective meaning subspace can be separated from the remaining factor structure by using the "markers" of the Osgood pan-cultural E-P-A dimensions as control traits in the resultant spatial configuration of scale vectors. The structure of the denotative subspace can then be analyzed independently. The only previous attempt to develop a quantitative rationale for this problem was made by Kuusinen (1969). From a matrix of correlations among 59 scales, he first computed a 12th order partial correlation matrix by successively partialling out the 12 E-P-A marker scales, and then derived a denotative structure by factoring this partial $(47 \times 47)$ correlation matrix. Miron (1969) criticized such a procedure on the grounds that the partial correlation technique would eliminate the greatest commonality of affect in accounting for the entire inter-scale relationships with respect to raters and rated concepts. Information on both the simultaneous co-existence of partialed structures of affective and denotative meaning components and their relative dominance for each scale in usage has been regarded as an important problem for contemporary psychosemantics (Osgood, 1964). The purpose of the present study is to present a new method based on a factor-analytic scheme for separating a global semantic space into affective and denotative subspaces given semantic differential ratings obtained for homogeneous sets of concepts.

\section{The Model}

In the case of measuring a given concept domain through the SD technique, let us assume that the SD scales to be used are obtained through a naturalistic elicitation procedure representing the universe of concept domain rele- 
vant traits (i.e., with high content validity in scale selection) (cf., Osgood, et al.. 1975; and Tzeng. 1976). The $m(=12)$ Osgood affective marker scales ( 4 scales to represent each E-P-A dimension) can be included as control variables in the final data collection of SD concept-onscale ratings.

If the resultant ratings are arranged in a three-mode data matrix $\mathbf{X}_{i j k}$ (subscript $i$ for scales from 1 to $n, j$ for concepts, and $k$ for subjects), the scale-by-scale cross-product matrix can then be obtained by computing across the concepts and subjects, and analyzed by a principal components solution based on Equations 1 and 2.

$$
\begin{aligned}
& i_{i}^{M_{i}}=i^{X}(j k) X_{i}^{\prime}, \\
& { }_{i}^{M_{i}}={ }_{i} \bar{A}_{i} Q_{i} \bar{A}_{i}^{\prime},
\end{aligned}
$$

In Equations 1 and $2 \vec{A}$ is the orthonormal matrix of the characteristic vectors of $\mathbf{M}$, and $\mathbf{Q}$ is the diagonal matrix of the characteristic values. Among all non-zero roots, let $r$ significant roots be identified as preceding the break from a steep slope to a gentler slope. These $r$ roots will be retained for subsequent analyses and represented by $Q_{r}$ (Tucker, 1966). This leaves the characteristic vectors ${ }_{i} \overline{\mathbf{A}}_{r}$ corresponding to $r$ roots.

The initial scale factor matrix $\mathbf{A}$ of $n$ scales by $r$ factors retained is obtained by Equation 3,

$$
A=\bar{A}_{r} Q_{r}^{1 / 2},
$$

and can be partitioned into two sub-domain matrices: $\mathbf{A}_{1}$, the marker domain (the $m$ E-P-A marker scales by $\boldsymbol{r}$ ), and $\mathbf{A}_{2}$, the non-marker domain (the $(n-m)$ other scales by $r$ ).

On the assumption that the Osgood E-P-A dimensions from a pan-cultural factorization of a heterogeneous concept domain are ubiquitous and can be recaptured even in indigenous cultural judgments of other homogeneous (e.g., personality) concepts, the $m$ markers in $\mathbf{A}_{1}$ should be three-dimensional with null loadings on the remaining $(r-3)$ factors. This is equivalent to finding a transformation matrix $\mathbf{T}_{r}^{*}$ such that

$$
A_{1}^{*}=A_{1} T_{r *}
$$

and $\mathbf{A}_{1}^{*}$ would be three-dimensional. This transformation matrix can be derived from solving for the eigenvalues and eigenvectors of the symmetric matrix $\mathbf{A}_{1}{ }^{\prime} \mathbf{A}_{1}$ :

$$
\begin{aligned}
\mathrm{A}_{1}{ }^{\prime} \mathrm{A}_{1} & =\mathrm{V} \Lambda \mathrm{v}^{\prime} \\
& =\left(\mathrm{v}_{1}: \mathrm{v}_{2}\right)\left[\begin{array}{ll}
\Lambda_{1} & 0 \\
0 & \Lambda_{2}
\end{array}\right]\left[\begin{array}{l}
\mathrm{v}_{1}^{\prime} \\
\mathrm{v}_{2}^{\prime}
\end{array}\right] .
\end{aligned}
$$

The number of significant roots in $\Lambda_{1}$ is expected to be three, under the assumption of affective universality, in correspondence to the Osgood pan-cultural affective space. The values of $\Lambda_{2}$ are expected to be zero or negligible. The characteristic vectors in Equation 5 are the transformation matrix that we are looking for. That is, let

$$
\begin{aligned}
\mathrm{T}_{\mathrm{r}^{*}}=\mathrm{V} & =\left(\mathrm{V}_{1}: \mathrm{V}_{2}\right) \\
& =\left(\mathrm{T}_{11}: \mathrm{T}_{22}\right) .
\end{aligned}
$$

This cosine matrix will then be applied to the initial factor structure as in Equation 7:

$$
\mathrm{A}^{*}=\mathrm{AT}_{\mathrm{r}} \text {, }
$$

or

$$
\begin{aligned}
{\left[\begin{array}{r}
A_{1}^{*} \\
A_{2}^{*}
\end{array}\right] } & =\left[\begin{array}{l}
A_{1} \\
A_{2}
\end{array}\right]\left(T_{11}: T_{22}\right) \\
& =\left[\begin{array}{cc}
A_{11}^{*} & A_{12}^{*} \\
A_{21}^{*} & A_{22}^{*}
\end{array}\right] .
\end{aligned}
$$


In the marker-scale domain, the following properties should be expected:

$$
\begin{aligned}
A_{1}^{*} A_{1}^{*} & =\left(T_{r^{*}}{ }^{\prime} A_{1}^{\prime}\right)\left(A_{1} T_{r^{*}}\right) \\
& =T_{r^{*}}{ }^{\prime}\left(A_{1}^{\prime} A_{1}\right) T_{r^{*}} \\
& =V^{\prime}\left(V \Lambda V^{\prime}\right) V=\Lambda, \\
A_{11}^{*}{ }^{\prime} A_{11}^{*} & =\left(A_{1} T_{11}\right)^{\prime}\left(A_{1} T_{11}\right) \\
& =T_{11}{ }^{\prime}\left(A_{1}^{\prime} A_{1}\right) T_{11} \\
& =V_{1}^{\prime}\left(V V^{\prime}\right) V_{1}=\Lambda, \\
A_{12}^{*} A_{12}^{*} & =\left(A_{1} T_{22}\right)^{\prime}\left(A_{1} T_{22}\right) \\
& =T_{22}{ }^{\prime}\left(A_{1}^{\prime} A_{1}\right) T_{22} \\
& =V_{2}^{\prime}\left(V \Lambda V^{\prime}\right) v_{2}=\Lambda_{2} .
\end{aligned}
$$

It is clear that when $\mathbf{T}_{r^{*}}$ is applied to $\mathbf{A}_{1}$, it differentiates the $n$ scale common principal component axes space into two subspaces-the affective space, $\mathbf{A}_{i 1}^{*}$, which is characterized by the structure of $m$ markers (corresponding to $T_{11}$ and $\Lambda_{1}$ ), and the non-affective space, $A_{12}^{*}$, which has null loadings from markers (corresponding to $T_{22}$ and $\Lambda_{2}$ ). (Note that, in some empirical research, there still may be some obvious variance in $\mathbf{A}_{12}^{*}$ from a particular marker when apparent interactions between concepts and scales occur.) Similarly, when $\mathbf{T}_{r *}$ is applied to $\mathbf{A}_{2}$, it rotates the non-marker scale factor matrix from the undifferentiated component space to the affective marker-defined space (as $\mathbf{A}_{21}^{*}$ by $\mathbf{T}_{11}$ ), and the remaining affect-free space (as $\mathbf{A}_{22}^{*}$ by $\mathbf{T}_{22}$ ).

From Equation 8, let
$A_{a}^{*}=\left[\begin{array}{c}A_{11}^{*} \\ \\ A_{21}^{*}\end{array}\right]$,

and

$$
A_{d}^{*}=\left[\begin{array}{c}
A_{12}^{*} \\
A_{22}^{*}
\end{array}\right] \text {. }
$$

Then $A_{a}^{*}$ is the factor structure of all $n$ SD scales in the E-P-A affective space, and $\mathbf{A}_{d}^{*}$ is the scale factor structure in the non-affective (denotative) semantic subspace. However, since $\mathbf{T}_{r}$ in Equations 7 and 8 acts only as a "filter" to divide the whole semantic space into two orthogonal subspaces, it is therefore desirable to rotate the factors to simple structure. That is, from the rotated structure of $A_{11}^{*}$ one should be able to evaluate the purity of the marker scales as E-P. $A$ affect markers when used in a homogeneous concept domain; and from the rotated structure of $\mathbf{A}_{22}^{*}$ one should be able to identify the character of non-affective dimensions when affective "contamination" is eliminated.

Let $\mathbf{A}_{11}^{*}$ and $\mathbf{A}_{22}^{*}$ be rotated by orthogonal or oblique transformation matrices $T_{11}^{*}$ and $T_{22}^{*}$ respectively:

$$
\mathrm{A}_{11}^{* *}=\mathrm{A}_{11}^{*} \mathrm{~T}_{11}^{*} \text {, }
$$

and

$$
\mathrm{A}_{22}^{* *}=\mathrm{A}_{22}^{*} \mathrm{~T}_{22}^{*}
$$

The two transformation matrices are then used in their respective affective or denotative subspace to rotate the non-marker scales onto the purified E-P-A dimensions: 


$$
\mathrm{A}_{21}^{* *}=\mathrm{A}_{21}^{*} \mathrm{~T}_{11}^{*}
$$

and also to rotate the $m$ marker scales onto the resultant denotative dimensions:

$$
A_{12}^{* *}=A_{12}^{*} T_{22}^{*}
$$

Therefore, from $\mathbf{A}_{21}^{* *}$ may be determined the amount of affective salience for each non-marker scale (i.e., the percent of sum of squares accounted for by E-P-A dimensions), and simultaneously from $\mathbf{A}_{12}^{* *}$ may be shown the degree of dominance of E-P-A markers on the "other" (denotative) dimensions (cf., Tzeng and May, 1975).

The transformation matrices $T_{11}^{*}$ and $T_{22}^{*}$ for $\mathbf{A}_{11}^{* *}$ and $\mathbf{A}_{22}^{* *}$ respectively can be augmented in the following fashion:

$\mathrm{T}_{\mathrm{r}^{* *}}=\left[\begin{array}{cc}\mathrm{T}_{11}^{*} & 0 \\ 0 & \mathrm{~T}_{22}^{*}\end{array}\right]$

The derivation of the final scale factor structure $A^{* *}$ can be summarized as follows. Let

$$
T_{r}^{*}=T_{r^{*}} T_{r * *}
$$

then

$$
\begin{aligned}
A^{* *} & ={ }_{i}{ }^{A} Q_{r}^{1 / 2} T_{r *} T_{r * *} \\
& ={ }_{i} \bar{A}_{r} Q^{1 / 2} T_{r}^{*} .
\end{aligned}
$$

\section{Analysis of a Set of Personality Ratings}

\section{Materials}

In the present study, data collected by Kuusinen (1969) on person perceptions were used, in cluding the ratings by 50 males of 25 personality concepts against $59 \mathrm{SD}$ scales. The scales were obtained from three sources: (1) 12 Finnish indigenous SD markers-four each to represent the E-P-A dimensions (Osgood et al.. 1975); (2) 16 scales taken from an earlier study by Ware of the structure of personality ratings (referred to in Miron and Osgood, 1966); and (3) 30 qualifiers, elicited directly from the same subject population, and their opposites, selected by the standard $\mathrm{H}$ (productivity index of each scale being elicited as a naturalistic response from different concepts) and Phi (degree of independence among elicited scales) procedures (Osgood, 1971). All subjects were middle class and living in an urban environment. Concepts rated were person types (including five ego concepts), six kinship concepts, five general person concepts, three well-known personalities, nine professions and trades, and two animal concepts. Scales are listed on the right of Table 1, with the first twelve being the E-P-A markers.

\section{Data Analysis}

For complete evaluation of the three-mode SD ratings (i.e., individuals, objects, and underlying psychosemantic criteria), the method described above was applied to the scale mode. The concept and subject modes factor matrices were obtained by principal components analyses with the concept mode solution rotated for interpretation by three rotational criteria: Varimax (Kaiser, 1958), Oblimax (Saunders, 1961), and the personal probability function solution (Tucker, 1970). In order to investigate the influence of affective and denotative semantic attribution in the factorial clustering of both the concept and subject modes, the inner core matrix was first derived through Tucker's (1966) 


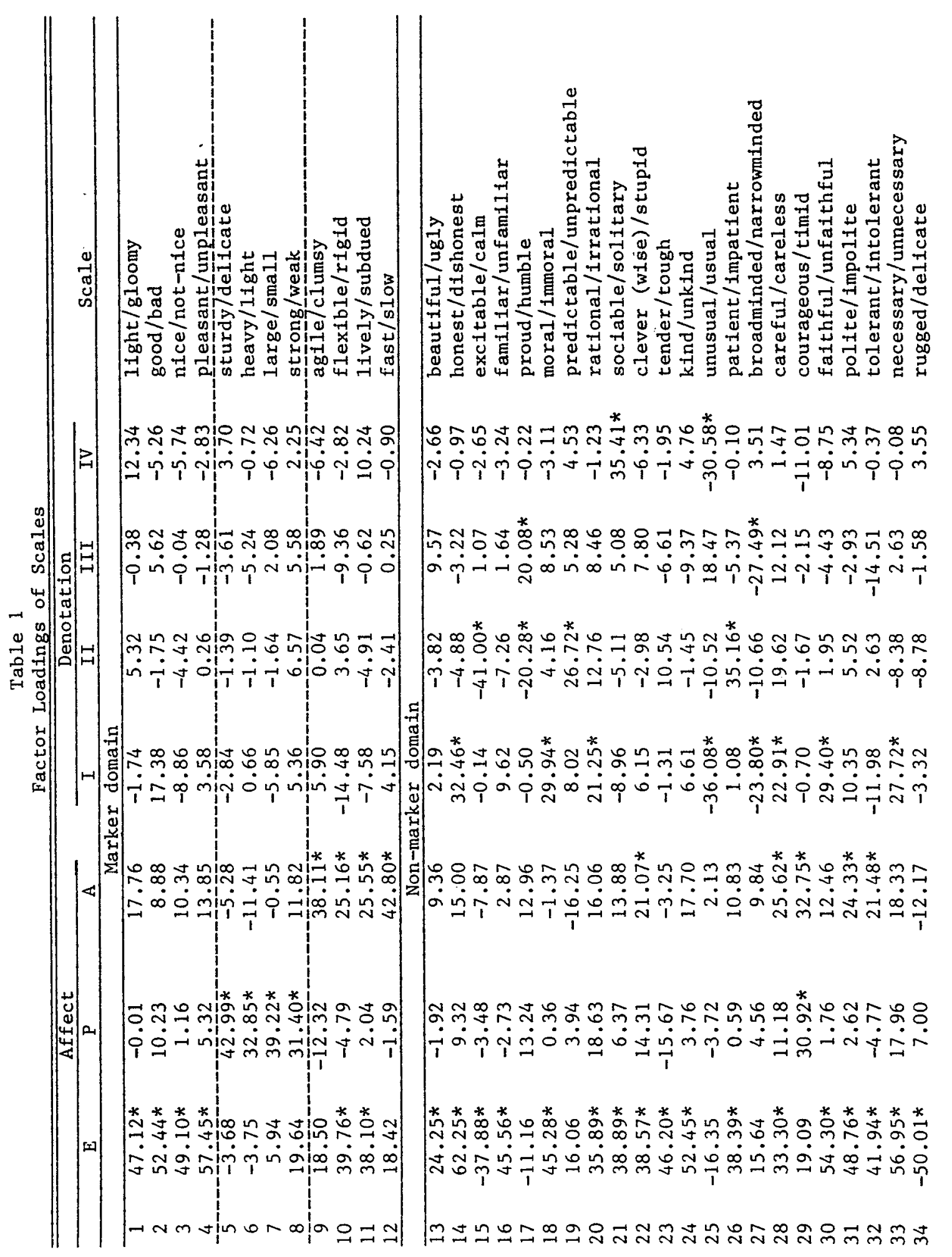




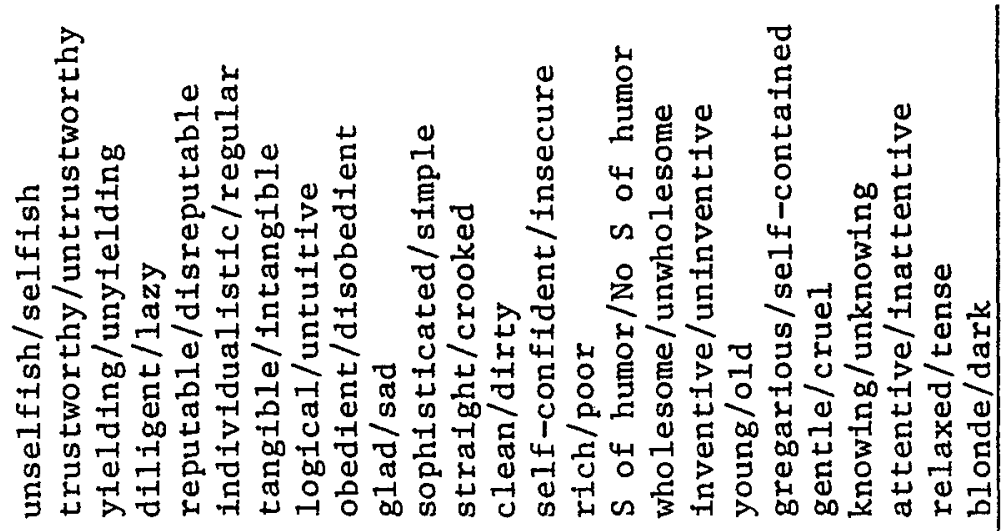

ㅊำ 꿍

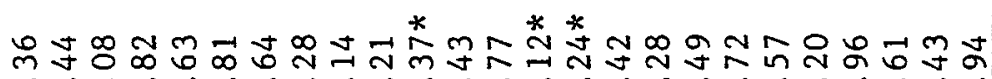
i

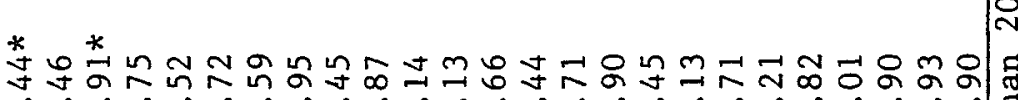
过过-ion

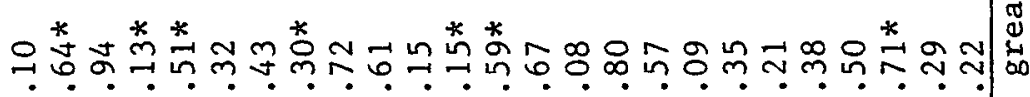

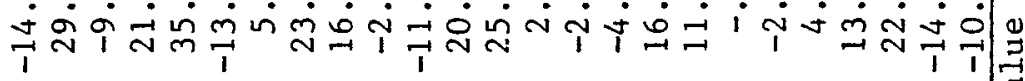

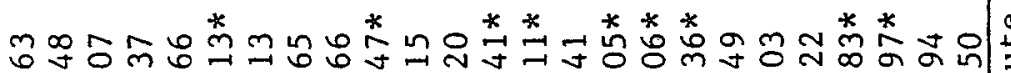

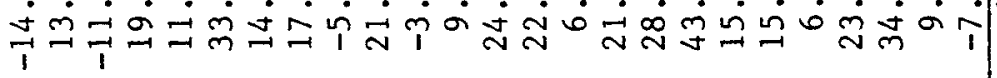

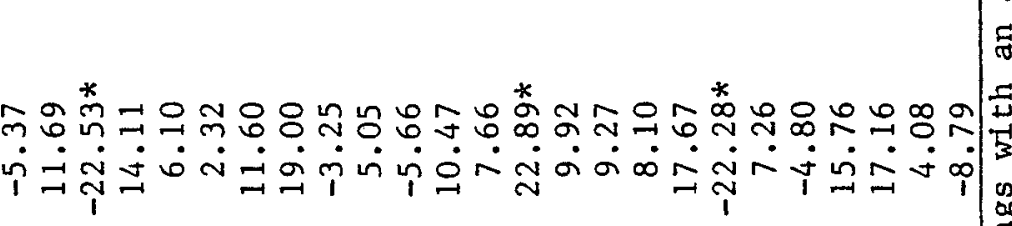

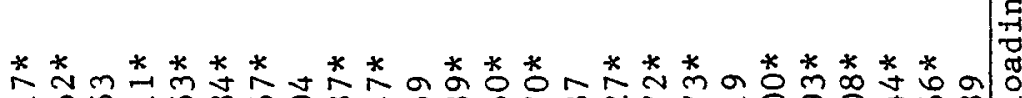

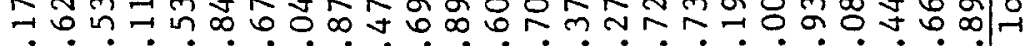

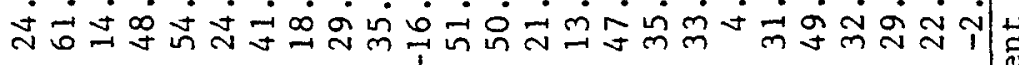

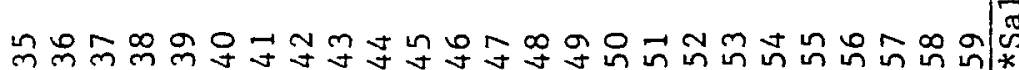


three-mode factor analytic model and then rotated in accordance with the rotations in the scale and concept modes. A graphical rotation was finally employed to maximize the idealized individual differences in the core matrix. The subject factor matrix was accordingly counterrotated.

\section{Results}

Semantic scale factors. The first ten roots from the principal components solution of the cross-product matrix of all 59 scales were $100,761,13,840,12,139,7,094,6,227,5,721$, $4,652,3,935,3,795$, and 3,472 . The first seven factors were retained, accounting for $65.35 \%$ of the total sums of squares. For the Affective marker domain, the eigenvalues of the sums of squares of the retained factor loadings of the 12 markers (from Equation 5) were 18,117, 6,057, $1,999,621,421,131$ and 92 . These values drop abruptly between the third and fourth, and taper off from the fourth. The first three roots accounted for $94.37 \%$ of the common sum of squares. This confirmed the assumption that the 12 pan-cultural E-P-A marker scales would be three-dimensional. The final rotated factor loading matrix $\left(\mathbf{A}^{* *}\right)$, from Equation 20, is shown in Table 1.

Affect. The most salient factor is the first, including four Evaluation and two Activity markers, with 36 out of 47 non-markers having a loading higher than an absolute value of 20.00 . These represent a general social evaluative meaning with such dominant traits as pleasant. good, nice, light (not gloomy), honest. trustworthy, reputable, faithful, kind, straight, clean, delicate, and gentle. The second factor is led by four Potency markers. Such traits as sturdy. large, heavy, strong, courageous, self-confident, and unyielding appear to characterize the Potency markers. The salient scales on the third factor include the four Activity markers-fast, agile, lively, and flexible, and other salient scales including inventive, attentive, individualistic. courageous, wholesome, careful, clean, and knowing. This factor is clearly associated with the active vs. non-active connotation of person perceptions.

The above factor loadings in $\mathbf{A}_{11}^{* *}$ seem to confirm that the 12 markers are rather distinct scales with simple loading patterns in recapturing the cross-cultural E-P-A constructs.

Denotation. The remaining four factors are in an orthogonal subspace. Among them, the first one, with high loadings for usual, reputable, honest, moral, trustworthy, faithful, necessary and clean, seems to characterize socially preferable (moral) behaviors and will be called a Morality dimension. The second factor is dominated by scales calm, patient, predictable, yielding. unselfish, and humble, vs. their opposites, excitable, impatient, unpredictable, unyielding. selfish, and proud. This dimension seems to describe a stable vs. unstable pattern of behavior and will be referred to as a Predictability dimension. The third factor has such salient scales as sophisticated, rich, narrow-minded, self-confident, and proud. on the one hand, and simple. poor, broad-minded, insecure, and humble, on the other-apparently reflecting polarity in social status. Therefore, choosing the subordinate pole, this factor will be called a Humility dimension. The last dimension seems to characterize individuals in interpersonal relationships as being either sociable, gregarious, usual, and regular, or solitary, self-contained, unusual and individualistic; this can be called a Sociability dimension.

These four factors, on which no affective markers have significant loadings, clearly reflect non-affective psychosemantic differentiation or description among the common personality concepts.

Concept factors. Four factors were retained, accounting for $55 \%$ of the total sum of squares of the inter-concept cross-product matrix computed across all scales and subjects. The salient factor loadings from the three rotational criteria are given in Table 2. The leading concepts for the first factor are MY IDEAL SELF, MYSELF AS A FRIEND, MYSELF, MY BEST FRIEND, MY MOTHER, MY SISTER, and MYSELF AS 
Table 2

Salient Concepts from Three Solutions

\begin{tabular}{|c|c|c|c|}
\hline Concept & $\begin{array}{l}\text { Varimax } \\
\text { Rotation }\end{array}$ & $\begin{array}{l}\text { Oblimax } \\
\text { Rotation }\end{array}$ & P.P.F.* \\
\hline \multicolumn{4}{|c|}{ Dimension 1} \\
\hline MY IDEAL SELF & 70.94 & 31.61 & .77 \\
\hline MYSELF AS FRIEND & 63.72 & 31.45 & .72 \\
\hline MYSELF & 60.88 & 22.65 & .71 \\
\hline MY BEST FRIEND & 60.76 & 39.23 & .69 \\
\hline MY MOTHER & 53.36 & 38.19 & .69 \\
\hline MY SISTER & 53.16 & 34.39 & .67 \\
\hline MYSELF AS A CHILD & 64.89 & 25.23 & .62 \\
\hline \multicolumn{4}{|c|}{ Dimension 2} \\
\hline POLICEMAN & 71.28 & 74.38 & .73 \\
\hline TEACHER & 61.42 & 62.33 & .66 \\
\hline POLITICIAN & 47.51 & 20.54 & .52 \\
\hline MY FATHER & 59.21 & 39.07 & .49 \\
\hline A PERSON I DISLIKE & 33.55 & 53.67 & .45 \\
\hline \multicolumn{4}{|c|}{ Dimension 3} \\
\hline \multicolumn{3}{|l|}{ MY UNCLE (FATHER'S } & .58 \\
\hline BROTHER) & 72.01 & 48.26 & .50 \\
\hline POLITICIAN & 21.85 & 26.81 & .40 \\
\hline MY COLLEAGUE & 58.21 & 33.94 & .37 \\
\hline MYSELF AS A FATHER & 58.17 & 26.36 & .32 \\
\hline MY FOREMAN & 39.76 & 22.46 & .30 \\
\hline MY IDEAL SELF & 43.29 & 11.09 & .17 \\
\hline \multicolumn{4}{|c|}{ Dimension 4} \\
\hline THIEF & 77.66 & 154.25 & .73 \\
\hline MURDERER & 77.51 & 130.04 & .57 \\
\hline ARTIST & 37.11 & 89.94 & .53 \\
\hline ALCOHOLIC & 48.02 & 84.33 & .44 \\
\hline POLITICIAN & 42.82 & 77.79 & .42 \\
\hline A PERSON I DISLIKE & 47.68 & 54.65 & .24 \\
\hline
\end{tabular}

A CHILD. These concepts are highly related to close friends and family and can be called the Admiration-Affection dimension. The second factor, dominated by POLICEMAN, TEACHER, and POLITICIAN, can be called the Popularity dimension. It is interesting to note that the concepts MY FATHER and A PERSON I DISLIKE are also correlated with public figures. Dimension 3, characterized by admir- able and socially respected roles and male personalities such as MEDICAL DOCTOR, MY UNCLE, POLITICIAN, MY COLLEAGUE, MYSELF AS A FATHER, MY FOREMAN and MY IDEAL SELF, can be labeled a Male Identification dimension. The last dimension is dominated by two outlaws, THIEF and MURDERER. It is quite interesting that ARTIST, ALCOHOLIC, POLITICIAN and A PERSON I 
DISLIKE are also salient on this. This factor can be called the Socially Undesirable dimenion.

Subject types. Three subject dimensions (or idealized subjects) were retained, accounting for $49 \%$ of the sum of squares of the inter-subject cross-product matrix. After counter-rotation in accordance with the graphic rotation for the inner core matrix, the first two subject dimensions appear somewhat correlated. But two clusters of subjects were clearly differentiable in the space. That is, Dimension 1 characterized effectively all 25 unmarried as well as 10 married subjects, while Dimension 2 characterized the other 12 married subjects. Dimension 3 is dominated by three married subjects. The differences in conceptions of personalities among these three idealized individuals are well-accounted for by the core matrix.

Core matrix. The resultant core matrix $(7 \times$ $4 \times 3$ ), after being counter-rotated with respect to the scale mode and the personal probability function solution in the concept mode, was strung out to form a Kronecker two-mode matrix, with the concept factors nested in the semantic scale components as the rows and the principal component subject factors as the columns. Graphic rotation was then applied to this three-dimensional idealized subject space and provided a simple structure as given in $\mathrm{Ta}$ ble 3 . Entries-loadings of three-mode $(7 \times 4 \times 3)$ factors-can be defined as the loadings of the four concept factors on the seven semantic meaning dimensions in the three idealized subjects' space. Therefore, entries in each column can be seen as the "judgments" of the four concepts factors against the seven psychosemantic components by each hypothetical subject; and only the ordinal properties of these concept/scale factorial interactions are used for in terpretation (Tzeng, 1975a).

For the first ideal subject, the Admiration-Affection and Popularity concept factors are extremely good $(+E)$, potent $(+P)$, active $(+A)$ and moral (+Morality), but not well differentiated on the other denotative components, except for Popularity being sophisticated and social, and Admiration-Affection being unsophis- ticated. Concepts related to the Male Identification dimension are seen as relatively potent $(+P)$, active $(+A)$, and sophisticated (-Humility), but neutral on Evaluation, Morality, Predictability, and Sociability. As to the Socially Undesirable concept factor, it has high positive loadings on Activity, but high negative loadings on Predictability and Humility.

The second idealized individual has a rather different semantic theory of personality. Concepts closely related to ego (i.e., the AdmirationAffection dimension) have only minor salience on Potency, Activity and Sociability. But concepts related to public affairs in the Popularity dimension are notably high on Evaluation and Activity and slightly positive on Morality. The Male Identification dimension is perceived, strangely enough, as being negative on all affective as well as denotative semantic components. In contrast, the social risk-taking personality concepts from the Socially Undesirable dimension are highly positive on all components. That is, they are perceived as not only extremely good $(+E)$, potent $(+P)$, and active $(+A)$, but also moral, predictable, sociable and sophisticated.

For idealized subject 3 , there seems no consistent pattern as to how meaning systems are being used to differentiate the four conceptual structures, except for Popularity being rated as good and active, and the Admiration-Affection dimension being relatively impotent $(-\mathrm{P})$ and passive (-A), but predictable (+Predictability).

\section{Discussion}

\section{Methodology for \\ Semantic Differential Ratings}

The SD technique has been widely used in judgments of diverse concept domains by diverse subject groups in various social and behavioral sciences. Unfortunately, many practitioners frequently used external (or arbitrary) criteria in selecting bi-polar scales without regard to their representativeness (content validity) of the universe of concept-relevant traits actually used by the subject population (Tzeng and Schultze, 
Table 3

Rotated Core Matrix

\begin{tabular}{|c|c|c|c|c|c|c|}
\hline \multirow[b]{2}{*}{ Scale Factor } & \multirow[b]{2}{*}{ ISC } & & \multicolumn{3}{|c|}{ Subject Factor } & \multirow[b]{2}{*}{ Concept Factor } \\
\hline & & ICO & 1 & 2 & 3 & \\
\hline \multicolumn{7}{|c|}{ Affective Space } \\
\hline \multirow{4}{*}{ Evaluation } & 1 & 1 & 181 & 1 & -1 & ADMIRATION-AFFECTION \\
\hline & 1 & 3 & 87 & 74 & 17 & POPULARITY \\
\hline & 1 & 3 & 2 & -138 & 7 & MALE IDENTIFICATION \\
\hline & 1 & 4 & 6 & 160 & 10 & SOCIALLY UNDESIRABLE \\
\hline \multirow{4}{*}{ Potency } & 2 & 1 & 23 & 14 & -10 & ADMIRATION-AFFECTION \\
\hline & 2 & 2 & 46 & 0 & 2 & POPULARITY \\
\hline & 2 & 3 & 36 & -13 & 3 & MALE IDENTIFICATION \\
\hline & 2 & 4 & -8 & 23 & 1 & SOCIALLY UNDESIRABLE \\
\hline \multirow{4}{*}{ Activity } & 3 & 1 & 79 & 14 & -9 & ADMIRATION-AFFECTION \\
\hline & 3 & 2 & 49 & 32 & 11 & POPULARITY \\
\hline & 3 & 3 & 15 & -39 & 7 & MALE IDENTIFICATION \\
\hline & 3 & 4 & 41 & 42 & 0 & SOCIALLY UNDESIRABLE \\
\hline \multicolumn{7}{|c|}{ Denotative Space } \\
\hline \multirow{4}{*}{ Morality } & $\overline{1}$ & 1 & 49 & 6 & 3 & ADMIRATION-AFFECTION \\
\hline & 1 & 2 & 53 & 16 & -2 & POPULARITY \\
\hline & 1 & 3 & 7 & -32 & -8 & MALE IDENTIFICATION \\
\hline & 1 & 4 & 2 & 50 & -3 & SOCIALLY UNDESIRABLE \\
\hline \multirow{4}{*}{ Predictability } & 2 & 1 & -1 & 0 & 8 & ADMIRATION-AFFECTION \\
\hline & 2 & 2 & -1 & 3 & 7 & POPULARITY \\
\hline & 2 & 3 & 3 & -31 & -7 & MALE IDENTIFICATION \\
\hline & 2 & 4 & -26 & 27 & 11 & SOCIALLY UNDES IRABLE \\
\hline \multirow{4}{*}{ Humility } & 3 & 1 & -12 & -4 & -7 & ADMIRATION-AFFECTION \\
\hline & 3 & 2 & 12 & -7 & 0 & POPULARITY \\
\hline & 4 & 4 & 18 & -6 & -1 & MALE IDENTIFICATION \\
\hline & 3 & 4 & -23 & 8 & -8 & SOCTALLY UNDESIRABLE \\
\hline \multirow{4}{*}{ Sociability } & 4 & 1 & -2 & 10 & 0 & ADMIRATION-AFFECTION \\
\hline & 4 & 2 & 10 & 4 & -2 & POPULARITY \\
\hline & 4 & 3 & 1 & -13 & 2 & MALE IDENTIFICATION \\
\hline & 4 & 4 & -2 & 17 & -4 & SOCIALLY UNDESIRABLE \\
\hline
\end{tabular}

1976). Quite often in the literature, SD ratings are analyzed blindly by the usual 2 -mode factor analysis for the purpose of measuring only affective components-Evaluation, Potency, and Activity. The standard factor-analytic procedure will only provide factor structures from the inter-vector relationships among the input scales; therefore, when scales used are neither relevant nor representative, the construct validity of the solutions may be questionable. This may be seen in the literature in two extremes: when all or part of the E-P-A dimensions are ob- 
tained, but due to the ubiquitous dominance of affective meaning systems in the entire factor structure (Miron, 1969), the other resultant dimensions following E-P.A are frequently identified as "secondary" affective dimensions; or when E-P-A are not "discovered", and the generality of Osgood's E-P-A structure is subsequently questioned. It is clear that standard factor analysis could not resolve the dispute over the non-affect versus multiple-affect components for a given homogeneous concept domain.

Of course, when the scales used are truly relevant (e.g., obtained through a naturalistic elicitation from the subject population (cf. Osgood et. al.. 1975, and Tzeng, 1976) standard factor analysis seems to be sufficient in providing valid psychosemantic structures (if the subject ratings are reliable). However, since it is impossible to include all relevant scales for subject ratings, selection becomes necessary. Unless the scales chosen are maximally representative (e.g., through some objective content analysis to derive scales with high frequency, productivity and diversity in usage across all subjects and concepts), the resultant factor solutions under usual procedures still do not serve the purpose of accurate description of human cognition with respect to the affect-nonaffect dispute. On the other hand, when the scales are truly relevant and maximally representative for a heterogeneous concept domain, a standard factor analysis procedure will likely produce indigeneous cultural E-P.A dimensions, with $P$ and $A$ being possibly less stable in magnitude of variance accounted for (cf., Osgood et al., 1975, p. 123). However, the orientation of these dimensions will not automatically coincide with that of the Osgood cross-culturally common affective space. Therefore, no direct cross-group and cross-cultural comparisons can be made on the affective meaning components and their influences in accounting for individual differences for a given concept domain. Furthermore. under indigenous factorizations, dimensions beyond $\mathrm{E}$ P-A are generally more obscure due to the massive dominance of affect, even though they may be psychologically real in the total differentiation of meanings (Osgood, 1969; Lane, 1973; Tzeng and May, 1975; Osgood et al.. 1975). Unfortunately, no technique has been available for their unique characterization.

With the present model and for a given set of SD scales selected objectively or even arbitrarily for a homogeneous concept domain and subject population, the Osgood E-P-A markers can always be included as control traits for both exploratory and confirmatory factor analyses for the entire semantic space. Under the notion of factorial validity (Brown, 1970), the resultant factor structures of $\mathbf{A}_{a}^{* *}$ (i.e., the factor loadings of both markers and non-markers in the first three dimensions) can be used to examine the convergent validity (Campbell, 1960; Campbell and Fiske, 1959) as well as reliability of Osgood's E-P-A psychological constructs. The present research has demonstrated that when the Finnish E-P-A markers, as derived from the pan-cultural factorization with respect to heterogeneous concepts from a teen-age male population, were used in a homogeneous personality descriptor vector space, they functioned well as the affective components for the Finnish male adults' personality conceptions.

In regard to the exploratory aspect of the model, the present methodology provides an independent and simultaneous evaluation of the other subspace in $\mathbf{A}_{22}^{* *}$ which is usually hidden in the undifferentiated, affectively dominant semantic space derived from the usual factor analysis solutions. The four dimensions identified in the present illustration appeared to be "affect-free" modes of distinguishing meanings of personalities. They were, therefore, defined as denotative meaning systems.

The characteristics and generality of the present factor solutions have been supported by an identical pattern of factor structures on our cross-cultural personality differential data from other culture/sex groups--both males and females from Japan, Finland, England, and Belgium (Tzeng, 1975b). The indigenous E-P-A marker scales had also been confirmed as highly reliable for ratings of other concept domains 
(e.g., natural concepts like POND and VALLEY and built concepts like CHAPEL and ELEVATOR) and subject populations (Hogenraad and Tzeng, 1976, Tzeng, 1975c).

Compared with the partialling technique (cf., Kuusinen, 1969), the present method will avoid the obvious problem of eliminating successively the covariance of E-P-A individual markers when their reliability and factorial validity are not known for a given homogeneous concept domain and/or a new subject population. If the purpose is merely to partial out the cross-cultural E-P-A dimensions, rather than markers. from an SD space, the present method will provide the exact data for this operation. Furthermore, beyond the solutions from the partialling technique, one is able to show the simultaneous coexistence and distribution of both affect and denotation for each scale, without excluding or disturbing the ubiquitous E-P-A saturations in human psychosemantic systems.

As stated before, the meanings of concepts reflect individual learning experiences and behavioral dispositions in the environment. The information of the concept factors, psychosemantic structures and their interactions for each homogeneous subject group with similar response patterns is extremely important for psychological explanation of individual differences on a particular issue (concept domain). The present methodology together with Tucker's three-mode factor analysis (Tucker, 1966), as demonstrated by the Finnish personality ratings, has high potential applicability to other subject and/or concept domains for better understanding of intra- and inter-cultural differences.

\section{Implications for Other Measurement Techniques}

The method developed here can be used in determination of the validity and reliability of psychological constructs in other multivariate research. It is obvious that given a data set from two domains of measurement variables (e.g., criteria or some known traits, and predictors) from an exploratory or cross-validation subject sam. ple, canonical correlation, multiple regression and usual factor analysis can be employed to identify their relationships. However, there are two possible weaknesses in their application: First, normalization of input variables, which is necessary in the first two approaches (in most cases, also in factor analysis, cf. Harman 1966) may not be desirable for practical purposes, due to insensitivity to significant constant differences and subject dispersions with respect to the entire set of variables. Second, final solutions of all three methods depend so much on the characteristics of the predictor variables domain that it is impossible to observe the criterion variables independently and also simultaneously. This is critical when the number of criterion variables is much smaller than the number of predictor variables. In this regard, the present research strategy, under which either correlation, covariance, or cross-products matrices could be used for input, may serve as an alternative method by following these steps: (1) identify the common dimensions for both prediction and criterion domains; (2) evaluate the structure of the criterion domain independently within the common space in order to verify or discover psychological constructs; (3) relate (or rotate) the predictor vectors to the resultant criterion subspace; and (4) evaluate the residual, unrelated subspace of the predictor domain. Following this procedure, the characteristics of correlation types of analyses for psychological constructs will be strengthened. Furthermore, by evaluation of structural relationships of the measurement variables in both domains with the resultant common dimensions across different subject samples or populations, the stability and reproducibility (reliability) of factor loadings of all measurement variables will be readily apparent.

\section{References}

Brown, F. G. Principles of educational and psychological testing. Hinsdale. Illinois: The Dryden Press, 1970.

Campbell. D. T. Recommendations for APA test standards regarding construct. trait or discriminant validity. American Psychologist. 1960, 15. 546-553. 
Campbell. D. T. and Fiske, D. W. Convergent and discriminant validation by the multi-trait-multimethod matrix. Psychological Bulletin. 1959. 56. $81 \cdot 105$.

Harman, H. H. Modern factor analysis. Chicago: University of Chicago Press. 1960.

Hogenraad, R. and Tzeng, O. C. S. Affective and denotative meaning systems in natural and built space conceptions. 1976 (in preparation).

Kaiser, H. F. The varimax criterion for analytic rotation in factor analysis. Psychometrika. 1958. 23. $187-200$.

Kuusinen, J. Affective and denotative structures of personality ratings. Journal of Personality and Social Psychology. 1969. 12.181-199.

Lane, S. T. M. Semantic differential scales for Portuguese speakers in Brazil. International Joumal of Psychology, 1973, 8. 147-152.

Miron, M. S. What is it that is being differentiated by the semantic differential? Journal of Personality and Social Psychology. 1969. 12, 189-193.

Miron, M. S. and Osgood, C. E. Language behavior: The multivariate structure of qualification. In $R$. Cattell (Ed.), Handbook of Multivariate Experimental Psychology. Chicago: Rand McNally and Company, 1966.

Osgood. C. E. Semantic differential technique in the comparative study of cultures. American $\mathrm{An}^{\text {- }}$. thropologists, 1964, 66(3), 171-200.

Osgood, C. E. On the whys and wherefores of E, P, and A. Journal of Personality and Social Psychology, 1969, 12(3), 194-199.

Osgood. C. E. Exploration in semantic space: A personal diary. The Journal of Social Issues. 1971 , 27(4), 5-64.

Osgood. C. E., May. W. H. and Miron. M. S. Cross cultural universals of aftective meaning. Urbana: University of Illinois Press. 1975.

Osgood. C. E.. Suci. G. H., and Tannenbaum, P. H. The measurement of meaning. Urbana: University of Illinois. 1957.

Saunders. D. R. The rationale for an "oblimax" methoa of transformation in factor analysis. Psychometrika. 1961, 26. 317-324.
Tucker. L. R. Some mathematical notes on threemode factor analysis. Psychometrika, 1966, 31 . 279-311.

Tucker. L. R. Fitting of factor analytic hyperplanes by a personal probability function. A report on techniques for investigation of structure of individual differences in psychological phenomena. Champaign: University of Illinois. Department of Psychology, September, 1970, 39-55.

Tzeng. O. C. S. Differentiation of affective and denotative meaning systems in personality ratings via three-mode factor analysis. Unpublished doctoral dissertation. University of Illinois at UrbanaCampaign, 1972. University Microfilms No. 7317.454. 278.

Tzeng. O. C. S. Differentiation of affective and denotative meaning systems and their influence in personality ratings. Journal of Personality and Social Psychologv. 1975. 32. 978-988. (a)

Tzeng. O. C. S. Differentiation of affective and denotative semantic subspace. Paper presented at the Conference on "Issues in Cross-cultural Research." The New York Academy of Science, New York City, October, 1975. (b)

Tzeng. O. C. S. Reliability and validity of semantic differential E-P-A markers for an American English representative sample. Psychological Reports. 1975. 37. 292. (c)

Tzeng. O. C. S. Application of semantic ditferential techniques in social and behavioral science research. The Consortium of International Studies Program. 1976 (in press).

Tzeng. O.C. S. and May, W. H. More than E. P. and $A$ in semantic differential scales: An answer to questions raised by Silvia T. M. Lane. International Journal of Psychology, 1975, 10(2), 101-117.

Tzeng. O. C. S. and Schultze. Q. An application of the semantic differential technique. A revien of literature. 1976 (in preparation).

\section{Author's Address}

Oliver C. S. Tzeng. Department of Psychology. Indiana University-Purdue University at Indianapolis. 1201 E. 38th St.. Indianapolis. IN 46205 